\title{
Fokker-Planck-Equation Approach to Flow Alignment in Liquid Crystals
}

\author{
Siegfried Hess * \\ Huygens Laboratorium, Rijksuniversiteit, Leiden \\ (Z. Naturforsch. 31 a, 1034-1037 [1976] ; received June 18, 1976)

\begin{abstract}
A nonlinear inhomogeneous relaxation equation for the alignment tensor is derived from a Fokker-Planck-equation for the orientational distribution function where torques exerted by a molecular field and by the gradient of the flow velocity are taken into account. Phenomenological coefficients characterizing the flow alignment in the isotropic and in the nematic phases are related to molecular parameters.
\end{abstract}

Theoretical results for the flow alignment in the isotropic and nematic phases of liquid crystals are presented. The point of departure is a FokkerPlanck equation for the orientational distribution function. The inter-particle correlation is taken into account through an internal molecular field. An equation of motion for the alignment tensor is derived. It is similar to a phenomenological equation previously obtained ${ }^{1}$ within the framework of irreversible thermodynamics. Now, however, the characteristic coefficients are related to "molecular" parameters and, in the nematic phase, to the magnitude of the order parameter. Applications to the isotropic phase (pretransitional behavior), to the nematic phase (dependence of the flow alignment angle on the order parameter), and the interrelation between flow alignment in both phases are discussed.

\section{Fokker-Plandk Equation}

A liquid composed of (effectively) axisymmetric particles is considered. The unit vector parallel to the figure axis of a particle is denoted by $\boldsymbol{u}$. The average $\langle\Psi\rangle$ of a function $\Psi=\Psi(\boldsymbol{u})$ is given by $\langle\Psi\rangle=\int \Psi^{\prime} f \mathrm{~d}^{2} u$ where $f=f(t, \boldsymbol{u})$ is the orientational probability distribution function normalized according to $\int f \mathrm{~d}^{2} u=1$.

The alignment associated with the birefringence in the isotropic phase and also with the spontaneous order in the nematic phase is the second rank tensor $\mathbf{a}=\langle\boldsymbol{\Phi}\rangle$ with $\boldsymbol{\Phi}=\xi \overline{\boldsymbol{u} \boldsymbol{u}}$. Here $\cdots$ indicates the symmetric traceless part of a tensor. A convenient

* On leave from Institut für Theoretische Physik der Universität Erlangen-Nürnberg, Erlangen, West Germany.

Reprint requests to Dr. S. Hess, Institut für Theoretische Physik der Universität Erlangen-Nürnberg, Glückstraße 6, D-8520 Erlangen. choice for the numerical factor $\xi$ is $\xi=\sqrt{\frac{15}{2}}$. This implies $\boldsymbol{\Phi}: \boldsymbol{\Phi}=5$. In the special case of an unaxial alignment, $\mathbf{a}$ can be written as $\mathbf{a}=\sqrt{\frac{3}{2}} \boldsymbol{a} \overline{\boldsymbol{n} \boldsymbol{n}}$ where the director $\boldsymbol{n}$ is a spacefixed unit vector. The scalar order parameter $a$ is given by $a=\sqrt{\frac{2}{3}} \xi\left\langle P_{2}(n \cdot u)\right\rangle$, $P_{2}(x)=\frac{3}{2}\left(x^{2}-\frac{1}{3}\right) ; S=\left\langle P_{2}\right\rangle$ is the order parameter used by Maier and Saupe ${ }^{2}$. An equation of motion for $\mathbf{a}$ will be derived from a Fokker-Planck-equation for $f$ to be stated below.

With the help of the differential operator $\mathcal{L}=$ $\boldsymbol{u} \times \partial / \partial \boldsymbol{u}$, the Fokker-Planck-equation assumed to govern $f$ is written as

$\partial f / \partial t+\boldsymbol{\omega} \cdot \mathcal{L} f-w \mathcal{L} \cdot[\mathcal{L} f-f \mathcal{L}(\mathbf{F}: \mathbf{\Phi})]=0$.

The second term describes the change of $f$ due to an average angular velocity $\omega$ of the molecules. In the following, $\omega=\frac{1}{2} \operatorname{rot} v$ is used where $\boldsymbol{v}$ is the average flow velocity of the liquid. The positive quantity $w$ is an orientational relaxation coefficient (often referred to as orientational diffusion coefficient). The term in Eq. (1) containing the tensor

$$
\mathbf{F}=T^{-1} T^{*} \mathbf{a}+(6 w)^{-1} \varkappa \mathbf{G}, \mathbf{G}=\overline{\nabla \boldsymbol{v}},
$$

is due to the torques exerted on the particles by an internal molecular field and by the velocity gradient $\mathbf{G} ; T$ is the temperature of the liquid. The characteristic temperature $T^{*}$ is defined such that the energy $\mathcal{H}$ associated with the molecular alignment is given by $\mathcal{H}=-k_{\mathrm{B}} T^{*} \mathbf{a}: \mathbf{\Phi} ; k_{\mathrm{B}}$ is the Boltzmann constant. For an unaxial alignment, $\mathbf{a}: \mathbf{\Phi}$ reduces to $\sqrt{5}$ a $P_{2}=5\left\langle P_{2}\right\rangle P_{2}$, and $\mathcal{H}$ becomes equivalent to the Maier-Saupe interaction ${ }^{2}$. The quantity $x$ occurring in $(2)$ is a characteristic coefficient which may have either sign.

For $T^{*}=0$, no internal molecular field present, Eq. (1) reduces to the Fokker-Planck-equation used previously ${ }^{3,4}$ to study the flow alignment of (dilute) 
colloidal solutions. In that case, $w$ and $\varkappa$ can be inferred from hydrodynamics. In particular, for a solvent with viscosity $\eta$ one has ${ }^{3-5}$

$$
w=k_{\mathrm{B}} T\left(8 \pi \eta R_{\mathrm{r}}{ }^{3}\right)^{-1}
$$

where $R_{\mathrm{r}}$ is an effective radius of a colloidal particle. For an ellipsoid of revolution with the ratio $p=R_{1} / R_{2}$ between the semiaxes $R_{1}$ (parallel to $\boldsymbol{u}$ ) and $R_{2}$, one has $\varkappa=\sqrt{\frac{6}{5}}\left(p^{2}-1\right) /\left(p^{2}+1\right)$. This expression for $\varkappa$ implies $\varkappa>0, \varkappa=0$, and $\varkappa<0$ for prolate, spherical and oblate particles, respectively. In the case of a pure liquid to be considered here, $w$ and $\varkappa$ are considered as given "molecular" parameters. The latter one is associated with the "nonsphericity" of the particles.

In the absence of a flow field $(\omega=0, \mathbf{G}=0)$, the stationary solution of Eq. (1) is

$$
f_{\text {eq }} \sim \exp \left\{\left(T^{*} / T\right) \mathbf{a}: \boldsymbol{\Phi}\right\} .
$$

Thus for an unaxial alignment, $f_{\mathrm{eq}}$ reduces to the distribution function used by Maier and Saupe ${ }^{2}$ to study the equilibrium properties (in particular, the phase transition isotropic-nematic) of liquid crystals.

\section{Relaxation Equation for the Alignment}

Next, it is indicated how an inhomogeneous relaxation equation (which turns out to be nonlinear) for the alignment tensor a can be obtained from the Fokker-Planck-equation. Multiplication of Eq. (1) by $\boldsymbol{\Phi}$ and integration over $\boldsymbol{u}$ yields an equation for the second rank tensor $\mathbf{a}$ which also contains a 4-rank alignment tensor $b \sim\langle\hat{\boldsymbol{u} \boldsymbol{u} \boldsymbol{u}}\rangle$. Similarly, the moment equation for $b$ contains terms which couple it with $\mathbf{a}$ and with a 6-rank alignment tensor. A closed system of equations for $a$ and $b$ is obtained by disregarding the tensors of rank $l \geqq 6$. Elimination of $b$ from these two equations subject to the assumption that terms involving products of $\mathbf{G}$ with $(\partial / \partial t) \mathbf{a}$ and $\boldsymbol{\omega} \times \mathbf{a}$ and terms nonlinear in $\mathbf{G}$ can be disregarded leads to

$$
\frac{\partial \mathbf{a}}{\partial t}-2 \widetilde{\omega \times \mathbf{a}}+\mathbf{J}+\tau_{\mathrm{a}}^{-1}\left(\boldsymbol{\Sigma}+\sqrt{2} \tau_{\mathrm{ap}} \tilde{\mathbf{G}}\right)=0,
$$

with

$$
\begin{gathered}
\boldsymbol { \Sigma } = A \mathbf { a } - \sqrt { 6 } B \longdiv { \mathbf { a } \cdot \mathbf { a } } + C _ { 1 } \mathbf { a }: \mathbf { a } \mathbf { a } + C _ { 2 } \longdiv { \mathbf { a } \cdot \mathbf { a } \cdot \mathbf { a } }, \\
A=\left(1-\frac{T^{*}}{T}\right), \quad B=\frac{1}{7} \sqrt{5} \frac{T^{*}}{T}, \quad C_{1}=\frac{13}{18} C, \\
C_{2}=\frac{5}{9} C, \quad C=C_{1}+\frac{1}{2} C_{2}=\frac{12}{49}\left(\frac{T^{*}}{T}\right)^{2},
\end{gathered}
$$

$$
\begin{aligned}
& \mathbf{J}=-\frac{7}{12} C \mathbf{a}:\left(\frac{\partial}{\partial t} \overline{\mathbf{a} \mathbf{a}}-4 \overline{\omega \times \overline{\mathbf{a}}}\right), \\
& \tilde{\mathbf{G}}=\mathbf{G}+\sqrt{6} B \frac{T}{T^{*}} \overline{\mathbf{a} \cdot \mathbf{G}} \\
& -\frac{35}{18} \frac{T}{T^{*}} C(\overline{\mathbf{a} \mathbf{a}}: \mathbf{G}+\overline{\mathbf{a} G}: \mathbf{a}) .
\end{aligned}
$$

The fact that no numerical coefficient occurs in the expression for $A$ is due to the choice $\xi=\sqrt{\frac{15}{2}}$ in the definition $\mathbf{a}=\xi\langle\overline{\boldsymbol{u} \boldsymbol{u}}\rangle$ for the alignment tensor. An equation of the form (3), with $\tilde{\mathbf{G}}$ replaced by its first term $\mathbf{G}=\overline{\nabla \boldsymbol{v}}$ and without the term $\mathbf{J}$, was obtained in Ref. ${ }^{1}$ where the "relaxation times" $\tau_{\text {a }}$ and $\tau_{\text {ap }}$ were considered as phenomenological coefficients. Now, they are related to the molecular parameters $w$ and $\varkappa$ by

$$
\tau_{\mathrm{a}}=(6 w)^{-1}, \quad \sqrt{2} \tau_{\mathrm{ap}}=-\varkappa \tau_{\mathrm{a}} .
$$

Notice that $\tau_{\text {ap }}$ can be expected to be negative for prolate particles. It should be mentioned that the neglect of the 4-rank alignment would have yielded an equation of the form (3) with $C=0$ in $(4-7)$.

\section{Isotropic Phase}

In the isotropic phase and for temperatures $T$ well above $T^{*}$, the terms nonlinear in the alignment can be disregarded. This linearized version of Eq. (3) is equivalent to the phenomenological equation obtained by de Gennes ${ }^{6}$. The relaxation time for the alignment is $\tau=\tau_{\mathrm{a}}\left(1-T^{*} / T\right)^{-1}$. For a stationary viscous flow with $|\omega| \tau \ll 1$, the flow alignment which leads to the flow birefringence is given by

$$
\mathbf{a}=-\sqrt{2} \tau_{\mathrm{ap}} A^{-1} \mathbf{G}=\varkappa \tau_{\mathrm{a}}\left(1-\frac{T^{*}}{T}\right)^{-1} \mathbf{G} .
$$

This pretransitional behavior, i. e. the dramatic in. crease of the flow alignment for $T \rightarrow T^{*}$ described by the factor $\left(1-T^{*} / T\right)^{-1}$ is typical for a mean field theory. It is also found experimentally ${ }^{7}$. The ratio $\mu / \nu$ between the phenomenological coefficient $\mu, \nu$ of de Gennes can now be related to the molecular parameter $\varkappa$ by

$$
\frac{\mu}{\nu}=-\frac{1}{\sqrt{15}} \frac{\tau_{\mathrm{ap}}}{\tau_{\mathrm{a}}}=\frac{1}{2} \sqrt{\frac{2}{15}} \% .
$$

The factor $V_{15}^{\frac{2}{15}}$ occurs because the alignment tensor used here is defined by $\mathbf{a}=\xi\langle\overline{\boldsymbol{u} \boldsymbol{u}}\rangle$ with $\xi=\sqrt{\frac{15}{2}}$ rather than $\xi=1$.

From the experimental data on MBBA as analysed by Clark $^{8}, \mu / \nu \approx 0.3$, and consequently $x=$ $\sqrt{3} \overline{0} \mu / v \approx 1.6$ is found. 


\section{Nematic Phase}

The nonlinear relaxation Eq. (3) has been studied in Reference ${ }^{1}$. In the absence of a flow field, it has the stable stationary solution $\mathbf{a}=\mathbf{a}_{\mathrm{eq}} \equiv \sqrt{\frac{3}{2}} e_{\mathrm{eq}} \overline{\boldsymbol{n} \boldsymbol{n}}$ with $a_{\mathrm{eq}} \neq 0$, corresponding to the nematic phase provided that the temperature $T$ is below the transition temperature $T_{\mathrm{K}}$ which is determined by $\delta_{\mathrm{K}}$ $=1-T^{*} / T_{\mathrm{K}}=\frac{2}{9} B^{2} / C$. For $T=T_{\mathrm{K}}$, one has $a_{\mathrm{eq}}=$ $\frac{2}{3} B / C$. With $B$ and $C$ given by $(5), \delta_{\mathrm{K}}=\frac{5}{54} \approx 0.09$, and $S=S_{\mathrm{K}} \equiv \frac{3}{7} \approx 0.43$ at $T=T_{\mathrm{K}}$ is obtained. Here $S$ is recalled as the Maier-Saupe order parameter which is related to $a_{\mathrm{eq}}$ by $a_{\mathrm{eq}}=\sqrt{5} S$. These values for $\delta_{\mathrm{K}}$ and $S_{\mathrm{K}}$ are very close to the results of Reference ${ }^{2}$. As is well known ${ }^{9}$, the theoretical value for $S$ is in good agreement with experimental data while the value for $\delta_{K}$ is too large by a factor of about 30 .

Next, a nonequilibrium situation is considered. In the nematic phase, $\mathbf{a}$ can be written as $\mathbf{a}=$ $\mathbf{a}_{\mathrm{eq}}+\tilde{\mathbf{a}}$ where $\mathbf{a}=\sqrt{\frac{3}{2}} a_{\mathrm{eq}} \overline{\boldsymbol{n} \boldsymbol{n}}$ is the equilibrium alignment with the constant equilibrium order parameter $a_{\text {eq }}$; the director $\boldsymbol{n}$ may be timedependent. The quantity $\tilde{\mathbf{a}}$ is the deviation of $\mathbf{a}$ from $\mathbf{a}_{\mathrm{eq}}$. Scalar multiplication of Eq. (3) by the unit vector $\boldsymbol{n}$ and the neglection of $\tilde{\mathbf{a}}$ everywhere except in $\boldsymbol{\Sigma}$ [notice that $\boldsymbol{\Sigma}\left(\mathbf{a}_{\mathrm{eq}}\right)=0$ ] leads to Leslie's constitutive law (e. g. see Refs. ${ }^{9}$ )

$\boldsymbol{n} \times \boldsymbol{h}=\boldsymbol{n} \times\left(\gamma_{1} N+\gamma_{2} \mathbf{G} \cdot \boldsymbol{n}\right)$,

$$
\boldsymbol{N}=\partial \boldsymbol{n} / \partial t-\boldsymbol{\omega} \times \boldsymbol{n},
$$

for the "molecular" field ${ }^{1} \boldsymbol{h}=-2 \sqrt{\frac{3}{2}} a_{\mathrm{eq}} P_{\mathrm{k}} \boldsymbol{\Sigma} \cdot \boldsymbol{n}$; $P_{\mathrm{k}}$ stands for $m^{-1} \varrho k_{\mathrm{B}} T ; m$ is the mass of a particle, $\varrho$ is the mass density of the liquid. The Leslic coefficients $\gamma_{1}$ and $\gamma_{2}$ are given by

$\gamma_{1}=3 a_{\mathrm{eq}}^{2} P_{\mathrm{k}} \tau_{\mathrm{a}}\left(1-\frac{1}{2} C a_{\mathrm{eq}}{ }^{2}\right)$,

$\gamma_{2}=2 \sqrt{3} a_{\mathrm{eq}} P_{\mathrm{k}} \tau_{\mathrm{ap}}\left(1+\frac{1}{2} a_{\mathrm{eq}} B \frac{T^{*}}{T}-\frac{59}{54} a_{\mathrm{eq}}{ }^{2} C \frac{T}{T^{*}}\right)$,

for $\tau_{\mathrm{a}}$ and $\tau_{\mathrm{ap}}$ see (8). If the terms containing $C$ in (11), (12) are neglected the dependence of $\gamma_{1}$ and $\gamma_{2}$ on the order parameter is of the form proposed by Helfrich ${ }^{10}$. In Ref. ${ }^{1}$ relations (11), (12) without the terms containing $B$ and $C$ have been stated.

\section{Flow Alignment Angle}

For a stationary viscous flow in the absence of external orienting fields, the director $\boldsymbol{n}$ lies in the plane determined by the flow velocity $\boldsymbol{v}$ and the direction of its gradient provided that the inequality $\left|\gamma_{2}\right|>\gamma_{1}$ is fulfilled ${ }^{9}$. The flow alignment angle $\chi$ between $\boldsymbol{n}$ and $\boldsymbol{v}$ is determined by

$$
\cos 2 \chi=-\gamma_{1} / \gamma_{2} \equiv \lambda^{-1} \text {. }
$$

Use of (5), (8) yields

$$
\begin{aligned}
\cos 2 \chi & =\lambda^{-1}(S) \equiv \sqrt{\frac{15}{2}} \frac{S\left(1-r S^{2}\right)}{\varkappa\left(1+s_{1} S-s_{2} S^{2}\right)}, \\
r & =\frac{30}{49}\left(\frac{T^{*}}{T}\right), \quad s_{1}=\frac{5}{14}, \quad s_{2}=\frac{590}{441} \frac{T^{*}}{T} ;
\end{aligned}
$$

$S$ is the Maier-Saupe order parameter. With $r \approx 0.6$, $s_{1} \approx 0.36, s_{2} \approx 1.34$, the ratio

$$
\left(1-r S^{2}\right)\left(1+s_{1} S-s_{2} S^{2}\right)^{-1}
$$

occurring in (13) is approximately equal to 0.95 and 1.07 for $S=0.3$ and $S=0.7$. Hence for $S \lesssim 0.7$, $\cos 2 \chi$ is approximately proportional to the order parameter $S$.

The expression $\lambda^{-1}=\lambda_{\mathrm{F}}{ }^{-1} \equiv 3 S(S+2 \alpha)^{-1}$ obtained by Forster ${ }^{11}$ is formally equivalent to (13) with $r=0, s_{2}=0, s_{1}=(2 \alpha)^{-1}, \varkappa=\sqrt{10} \alpha$. The last equality, however, does not seem to be meaningful. The molecular parameter ${ }^{11} \alpha$ is defined such that $\varkappa \sim \alpha^{-1}$ can be expected (e.g. one has $\varkappa=0$ and $\alpha^{-1}=0$ for a spherical particle).

For $\left|\gamma_{2}\right|<\gamma_{1}$, no spatially homogeneous solution for the director $\boldsymbol{n}$ can be found which is stationary. According to (8), (12) and (13), this case is more likely to be encountered for molecules with small $\varkappa$, i. e. with small nonsphericity.

\section{Interrelation Between Flow Alignment in the Isotropic and in the Nematic Phases}

Comparison of (13) and (9) shows that the molecular nonsphericity parameter $\varkappa$ is of crucial importance both for the flow birefringence in the isotropic phase and for the flow alignment angle in the nematic phase. With $\varkappa=\sqrt{30} \mu / v$, the relation

$\lambda=R(\mu / v)$,

$R=2 S^{-1}\left(1+s_{1} S-s_{2} S^{2}\right)\left(1-r S^{2}\right)^{-1} \approx 2 S^{-1}(15)$

is found. The ratio $\mu / v$ can be inferred from measurements in the isotropic phase; $\lambda$ is a property characteristic for the nematic phase. The phenomenological law formulated in Ref. ${ }^{1}$ implies (14) with $R=2 S^{-1}$. Following a different line of reasoning, Clark $^{8}$ has suggested the existence of the relation (14) valid at the transition temperature $T_{\mathrm{K}}$ with 
$R=5$. From (15), $R \approx 4.6$ is obtained for $T=T_{\mathrm{K}}$. Within the experimental error, both these values for $R$ are in good agreement with the experimental data on MBBA as analysed in Reference ${ }^{8}$. Further measurements are desirable.

The Fokker-Planck-equation approach presented here gives some insight into the molecular processes underlying the relaxation of the alignment and the flow alignment. A relation between the "molecular" parameters $w, x$ and the intermolecular interaction potential, however, is still lacking.

1 S. Hess, Z. Naturforsch. 30 a, 728; 1224 [1975].

2 W. Maier and A. Saupe, Z. Naturforsch. 13 a, 564 [1958]; 14 a, 882 [1959]; 15 a, 287 [1960].

3 A. Peterlin and H. A. Stuart, Hand- und Jahrbuch d. Chem. Phys., eds. A. Eucken and K. L. Wolf, 8 IB, Leipzig [1943].

4 V. N. Pokrovskii, Soviet Phys. Uspekhi 14, 737 [1972].

5 S. Hess, Physica 74, 277 [1974].

6 P. G. de Gennes, Phys. Letters 30 A, 454 [1969].

\section{Acknowledgements}

I highly appreciate that Prof. J. J. M. Beenakker and Prof. H. F. P. Knaap have made it possible for me to again spend some time in the stimulating atmosphere of the Molecular Physics Group of the University of Leiden.

This work is part of the research program of the "Stichting voor Fundamenteel Onderzoek der Materie (F.O.M.)" and has been made possible by financial support from the "Nederlandse Organisatie voor Zuiver Wetenschappelijk Onderzoek (Z.W.O.)".

7 P. Martinoty, S. Candau, and F. Debeauvais, Phys. Rev. Letters 27, 1123 [1971].

8 N. A. Clark, Phys. Letters 46 A, 171 [1973].

9 M. J. Stephen and J. P. Straley, Rev. Mod. Phys. 46, 617 [1974] ; P. G. de Gennes, The Physics of Liquid Crystals, Clarendon Press, Oxford 1974.

10 W. Helfrich, J. Chem. Phys. 56, 3187 [1972].

11 D. Forster, Phys. Rev. Letters 32, 1161 [1974]. 